\title{
Shikumi Lab: origami-inspired positioning and fixing method of photomask for making various holographic optical elements (Withdrawal Notice)
}

Kyohei Yoshikawa, Kunio Sakamoto

Kyohei Yoshikawa, Kunio Sakamoto, "Shikumi Lab: origami-inspired positioning and fixing method of photomask for making various holographic optical elements (Withdrawal Notice)," Proc. SPIE 10818, Holography, Diffractive Optics, and Applications VIII, 108181F (2 November 2018); doi: $10.1117 / 12.2500026$ 


\section{Shikumi Lab: origami-inspired positioning and fixing method of photomask for making various holographic optical elements (Withdrawal Notice)}

Kyohei Yoshikawa, and Kunio Sakamoto

Konan Univ. (Japan)

Proc. SPIE 10818, 108181F (2018)

Online Publication Date: 2 November 2018

Withdrawn from Publication: 4 February 2019

Conference Date: 11 October-13 October 2018

Conference Location: Beijing, China

Conference Title: Holography, Diffractive Optics, and Applications VIII

Conference Chairs: Yunlong Sheng, Chongxiu Yu, Changhe Zhou

Publisher's Note: This manuscript, originally published on 2 November 2018, has been withdrawn by the publisher for editorial reasons. 